\title{
¿Por qué no se vacunan nuestras pacientes? Motivos por los que las pacientes fuera de los programas de vacunación sistemática, con infección por el virus del Papiloma Humano, deciden decir no a la vacuna
}

\author{
Gloria Cordeiro V. ${ }^{1}$, Sonia Pérez $C{ }^{2}{ }^{2}$, Amparo Iñarrea $F^{3}$, Daniel Simón V. ${ }^{a}$, \\ Consuelo Reboredo R. ${ }^{b}$, Emilio Couceiro N. ${ }^{4}$, Carlos Ramón y Cajal ${ }^{5}$. \\ 1 Ginecología y Obstetricia, ${ }^{2}$ Microbiología, ${ }^{3}$ Patología Cervical, ${ }^{4}$ Jefe de Sección Obstetricia, ${ }^{5}$ Jefe de Servicio \\ Obstetricia y Ginecología. Complejo Universitario Hospitalario de Vigo. Vigo, España.
}

$\mathrm{a}_{\text {Enfermera, Servicio de Urgencias, }}{ }^{\mathrm{b}}$ Enfermera, Patología Cervical. Complejo Hospitalario Universitario de Vigo

\section{RESUMEN}

Objetivo: Conocer los motivos de la no vacunación en mujeres fuera de los programas de vacunación sistemática. Metodología: Se evaluaron mediante cuestionario 226 mujeres con infección por VPH. A todas ellas se les había informado de su patología, del VPH y de la vacuna y se les había recomendado el uso del preservativo previamente. Resultados: El $66,7 \%$ tenían pareja estable; $75 \%$ utilizaban preservativo. La edad de inicio de relaciones sexuales fue 17,8 años y el 47,3\% de las pacientes habían tenido 5 ó más parejas a lo largo de su vida. El $72 \%$ conocía su infección por VPH y el $48,8 \%$ su lesión. El $63,7 \%$ no se vacunó: principalmente rechazaron la vacuna por el precio $(49,5 \%)$, información insuficiente $(18,7 \%)$ u otras causas como la disparidad de opiniones entre los médicos que la atendieron (15\%). Conclusiones: El coste de la vacuna y el conocimiento sobre el VPH son determinantes para la aceptación de la vacunación.

\section{PALABRAS CLAVE: Vacunas Virus Papiloma Humano, adulto, conocimiento, actitud, neoplasia intraepitelial cervical, recomendación}

\section{SUMMARY}

Objective: The aim of the study was to determine the reasons for non-vaccination in women outside the routine immunization programs. Method: There were evaluated by questionnaire 226 women with HPV infection. All of them had been informed of their disease and type of HPV infected by. We all had recommended the vaccine and the condom use previously. Results: $66.7 \%$ had a steady partner, $75 \%$ used condoms. The age of first sexual relationship was 17.8 years old and $47.3 \%$ of patients had 5 or more partners during their lifetime. $72 \%$ knew their HPV infection and $48.8 \%$ knew their injury. $63.7 \%$ were not vaccinated: they rejected the vaccine mainly for the price $(49.5 \%)$, insufficient information $(18.7 \%)$ or other causes such as primary care opposite point of view (15\%). Conclusions: The prize of the vaccine and the HPV knowledge are crucial to the acceptance of vaccination.

KEY WORDS: Papilloma virus vaccines, adult, knowledge, attitude, cervical intraepitelial neoplasia, recommendation 


\section{INTRODUCCIÓN}

El cáncer de cuello uterino es el más común en las mujeres menores de 45 años, situándose entre los tres primeros cánceres en el $90 \%$ de los países (1). El virus del papiloma Humano (VPH) es el principal agente etiológico de las lesiones precancerosas y del cáncer de cérvix $(2,3)$.

Las dos vacunas frente al cáncer de cérvix actualmente disponibles en España están financiadas para su administración en al menos una cohorte de niñas entre 11 y 14 años dentro de los programas de vacunación sistemática de cada Comunidad Autónoma (4).

Diferentes estudios $(5,6,7)$ en mujeres mayores de 25 años han confirmado la elevada eficacia preventiva de la vacuna, sin embargo no se recomienda la vacunación sistemática ya que no resulta coste - efectiva.

El riesgo significativo de adquirir nuevas infecciones por el VPH y el riesgo de mayor persistencia viral aumentan con la edad. Las mujeres adultas con infección por alguno de los tipos vacunales obtienen alta protección frente al resto de tipos, y las mujeres con infecciones por VPH previas y aclaramiento viral pueden protegerse de la reinfección y reactivación (8).

El objetivo del estudio fue conocer los motivos de la no vacunación en mujeres fuera de los programas de vacunación sistemática.

\section{SUJETOS Y MÉTODO}

Se incluyeron 226 mujeres (edad media de 29,7 años) que acudieron a la consulta de patología cervical del Complejo Hospitalario Universitario de Vigo entre Marzo 2013 y Febrero 2014. Todas ellas habían sido diagnosticadas de alteración citológica e infección por VPH, y llegaron a nuestra consulta a través del programa de cribado oportunista de lesiones cervicales, realizado mediante citología en las consultas de atención primaria o en alguna de las consultas de ginecología. Una vez que acuden a nuestra consulta son seguidas con control citológico - colposcópico y PCR para la detección del ADN viral, y son tratadas en los casos necesarios, hasta que puedan volver al cribado realizado por atención primaria.

Todas las pacientes habían asistido a la consulta por lo menos una vez previamente a la realización de la encuesta y habían sido informadas sobre su patología cervical y el VPH, a todas se les había recomendado la vacuna, el uso del preservativo y el abandono del hábito tabáquico en el caso de pacientes fumadoras. Todas fueron seguidas en la consulta mediante control citológico y de PCR para conocer la eliminación del virus y en cada consulta se informó de su situación y de la eliminación del virus en los casos oportunos.

Se las evaluó mediante una encuesta que incluyó 10 preguntas estructuradas en dos bloques según el contenido. El primer bloque con 6 preguntas sobre los hábitos y vida sexual de las pacientes y el segundo bloque sobre la infección por VPH y la vacuna.

Todas las pacientes firmaron un consentimiento informado para su inclusión en el estudio. Para todas las variables cuantitativas, se calculó la media y desviación estándar.

\section{RESULTADOS}

El $66,3 \%(n=152)$ de las pacientes tenían pareja estable y el $76,1 \%(n=172)$ usaban el preservativo como método anticonceptivo, de ellas 139 lo usaban como único método, 30 pacientes combinado con anticoncepción hormonal y 3 mujeres lo utilizaban combinado con el dispositivo intrauterino (DIU) (Figura 1). La media de edad de inicio de las relaciones sexuales fue 17,7 años y el $47,3 \%(n=104)$ había tenido 5 ó más parejas a lo largo de su vida.

En cuanto al nivel de conocimiento sobre la patología, el 74,3\% ( $n=168)$ sabía que padecía una infección por VPH y el 59,3\% ( $n=134)$ conocía el nombre de la lesión cervical que padecían.

No se vacunaron 134 mujeres. Las principales razones para la no vacunación fueron el coste de la vacuna $(54,5 \%, n=73)$ e información insuficiente $(14,9 \%, n=20)$. El 7,5\% $(n=10)$ de las pacientes tenía miedo a los efectos secundarios y el $6 \%(n=8)$ pensaba que tras haber recibido tratamiento ya no la necesitaba. En un 12,7\% $(n=17)$ de las pacientes las razones eran diversas principalmente la discordancia de información entre el médico de atención primaria y el ginecólogo (Tabla I). Para tomar la decisión de vacunarse, el hecho de conocer el agente causal responsable de la infección (VPH) no influyó en la elección (Tabla II).

\section{DISCUSIÓN}

Según datos publicados en la literatura el haber tenido más de una pareja sexual a lo largo de la vida, la edad temprana de inicio de relaciones sexuales y los antecedentes de infecciones de transmisión sexual, se asocian a un mayor riesgo de infección por VPH (9). A partir de los datos obtenidos en nuestro estudio sabemos que la media de edad de inicio de las relaciones sexuales de las mujeres encuestadas fue 17,7 años y que aproximadamente la mitad $(n=104)$ de las pacientes habían tenido 5 ó más parejas a lo largo de su vida. 


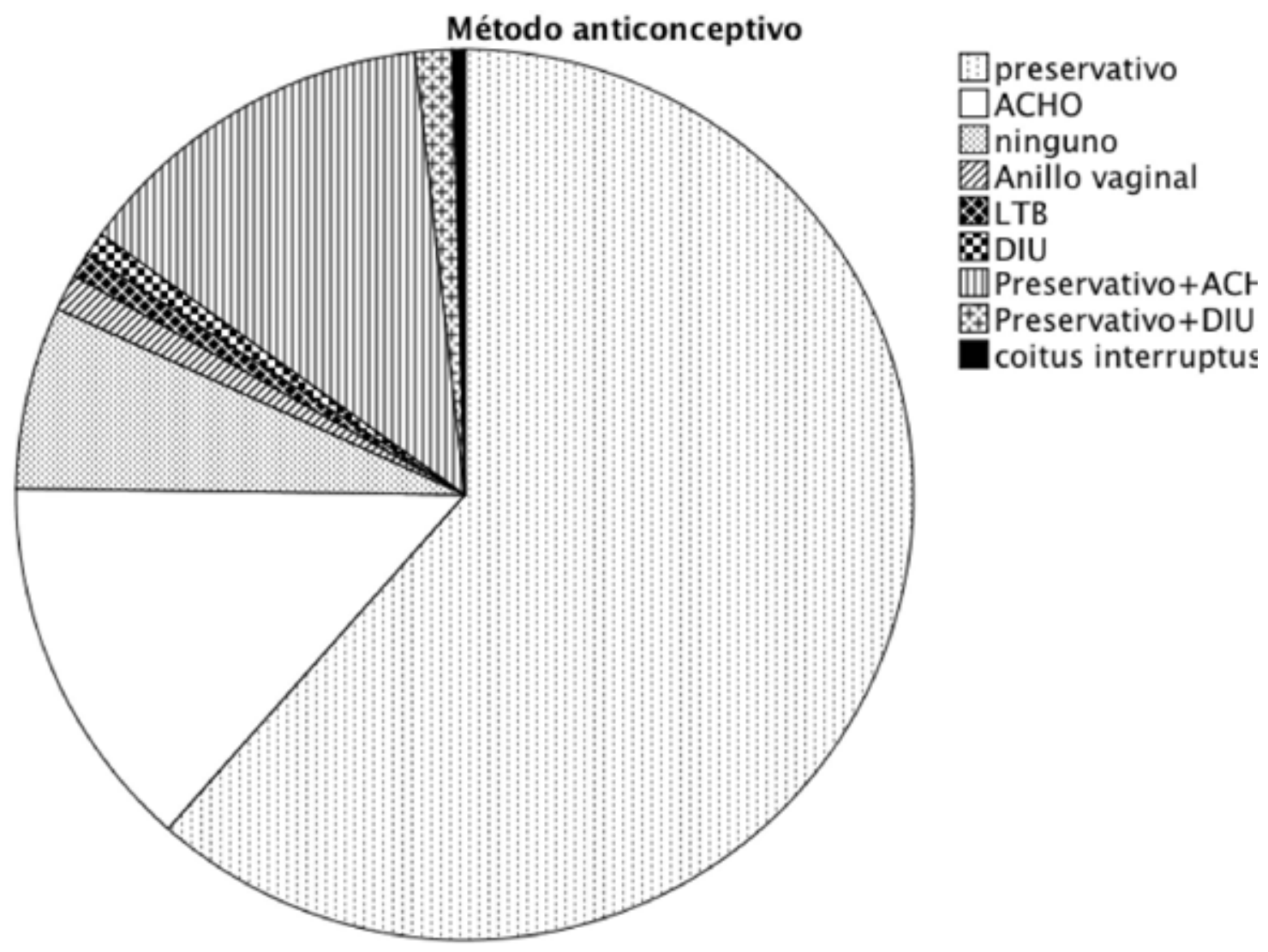

Figura 1. Método anticonceptivo utilizado por las pacientes.

Tabla I

RAZONES PARA LA NO VACUNACIÓN

\begin{tabular}{llcr}
\hline Vacunación & & Frecuencia & $\begin{array}{r}\text { Porcentaje } \\
\text { válido (\%) }\end{array}$ \\
\hline Pacientes no vacunadas & No me han hablado de vacuna & 20 & 14,9 \\
& Coste & 73 & 54,5 \\
& Miedo a efectos secundarios & 10 & 7,5 \\
& No creo en su eficacia & 6 & 6,5 \\
& Ya me he curado y no la necesito & 8 & 12,7 \\
& Otros & 17 & 100,0 \\
\hline Pacientes vacunadas & Total & 134 & 92 \\
\hline Total & Total & 226 & \\
\hline
\end{tabular}


Tabla II

VACUNACIÓN EN FUNCIÓN DEL CONOCIMIENTO DEL VPH

\begin{tabular}{lcccc}
\hline & & \multicolumn{2}{c}{ Vacunada } & Total \\
\hline Conoce agente causal de infección (VPH) & si & 70 & 98 & 168 \\
Total & no & 22 & 36 & 58 \\
\hline
\end{tabular}

Este virus es altamente contagioso y se ha estimado que la probabilidad de transmisión del VPH tras mantener relaciones sexuales sin preservativo puede ser del 40 al 60\% (10). A pesar de proporcionar una alta protección frente la infección por HPV, existen estudios (11) afirmando que el uso de preservativo no proporciona una protección completa frente a la transmisión de este virus. ¿Qué podemos por tanto ofrecer a nuestras pacientes? El uso del preservativo forma parte de la estrategia preventiva seguida en la consulta de patología cervical para potenciar la eliminación del virus y prevenir la reinfección; de este modo la mayoría $(76,1 \%)$ de las pacientes que habían sido previamente informadas sobre su uso lo utilizaban como método anticonceptivo.

Con la vacunación las mujeres adultas con infección por alguno de los tipos vacunales obtienen protección frente al resto de tipos, y las mujeres con infecciones por VPH previas y aclaramiento viral pueden protegerse de la reinfección y reactivación (8). Aproximadamente el $80 \%$ de la población femenina está expuesta a VPH en algún momento de su vida, pero la infección suele ser transitoria, con un aclaramiento viral en los 12-24 primeros meses en el $70-90 \%$ de las pacientes (11). El riesgo acumulado durante 5 años de tener una infección por VPH es de $42,5 \%$ en mujeres entre 15 y 19 años, y este riesgo disminuye a un $30 \%$ entre las mujeres de 25 y 29 años, siendo todavía menor (22\%) en las mujeres de edades comprendidas entre 30 y 44 años. Sin embargo se ha observado un pico de incidencia en la cuarta y quinta década de la vida sin conocerse la causa exacta de por qué ocurre: nuevas infecciones, una reactivación viral, el descenso en la inmunidad o mecanismos que todavía desconocemos (5), por lo tanto consideramos que la vacuna en mujeres fuera de los programas de vacunación sistemática podría ser una buena alternativa en estas mujeres cuyo riesgo se incrementa.

La principal razón para la no vacunación en el estudio fue el coste de la vacuna, una de cada dos mujeres encuestadas no se vacunaron por el precio de la misma. En un trabajo (1) publicado en 2011 en Human Vaccines los autores discuten los siguientes puntos acerca de la baja aceptación de la vacuna en mujeres entre 26 y 45 años: dudas sobre la eficacia y la seguridad, la actitud de las autoridades sanitarias españolas, el precio de la vacuna, el papel de los medios de comunicación y los grupos "antivacuna", así como el conocimiento del ginecólogo en este campo. Como conclusión los autores afirman que la amplia cobertura de la vacuna contra el VPH se lograría con el esfuerzo coordinado de todos los órganos que participan en el proceso de vacunación. Aplicado a los datos presentados se conseguiría un 49,5\% más de vacunación con la cobertura y financiación de la vacuna en las pacientes con patología cervical fuera de los programas de vacunación sistemática.

En este estudio la falta de información constituye la segunda causa de la no vacunación (14,9\%). Todas las participantes en la encuesta fueron mujeres que acudieron previamente a la consulta de patología cervical, en la cual recibieron información sobre el VPH y sus posibles consecuencias, así como explicaciones sobre sus patologías respectivas. Por lo tanto esto nos hace plantearnos que se produce un fallo en la cadena de comunicación, y en la comprensión de la paciente, ya que un $51,2 \%$ desconocía el tipo de lesión cervical que presentaba tras haber sido informada en una consulta previa. En otros datos comprobamos que una mayor educación sobre el VPH aumentó la aceptabilidad de la vacuna (12). Habiendo demostrado que un público informado aceptará más probablemente la vacunación, y que los médicos y personal sanitario son las personas de mayor confianza para difundir esta evidencia, es fundamental la actitud en este colectivo (13).

El miedo a las reacciones adversas constituye la razón de rechazo de la vacuna en el $7,5 \%$ de los casos, sin embargo el perfil de seguridad de la vacuna en mujeres de edad media es similar al de las mujeres menores de 24 años. Los efectos secundarios más frecuentes son malestar, fatiga y dolor local en el punto de inyección. Éste último ha sido más frecuentemente descrito en el grupo de mujeres de entre 24 y 45 años (14).

Los estudios existentes en la actualidad apuntan a una menor rentabilidad de la vacuna a medida que aumenta la edad de la población, y ésta es la 
principal razón por la que la vacunación a mujeres mayores de 24 no forma parte de las prioridades en las recomendaciones de salud pública relacionadas con el VPH. Sin embargo, las mujeres sexualmente activas de todas las edades podrían optar al beneficio de la vacunación y poder tener esta opción como una herramienta más en la lucha contra la patología cervical (14).

Más de la mitad de las pacientes $(59,3 \%)$ no se vacunaron tras las recomendaciones dadas, analizando las principales razones para la no vacunación tenemos el coste de la vacuna en primer lugar y la información insuficiente, como acabamos de comentar, el miedo a los efectos secundarios, la creencia de que tras haber recibido tratamiento ya no la necesitaban y diversas razones más como la discordancia de información entre el ginecólogo y el médico de atención primaria o entre los propios especialistas. Una encuesta de opinión (8) dirigida a ginecólogos españoles especializados en patología cervical demuestra que existe un desconocimiento sobre los aspectos importantes de la vacuna frente al cáncer de cérvix en mujeres fuera de los programas de vacunación sistemática con patología cervical. Por otro lado la recomendación del médico de atención primaria o especialista ha demostrado ser a nivel internacional el motivo más influyente para la aceptación de la vacuna (15-18).

A pesar de que sabemos que estrategias preventivas como la vacunación o el uso del preservativo no pueden asegurar la prevención de nuevas infecciones por VPH en las mujeres que atendemos en la consulta de patología cervical, creemos necesario ofrecerla a la población que atendemos, por su riesgo de adquirir nuevas infecciones.

Creemos que la importancia de este estudio es doble. Por un lado muestra datos actuales de la situación de las mujeres en España con patología cervical, información sobre sus hábitos sexuales y conductas de riesgo para la infección por el VPH, mientras que por otro abre las puertas a futuras líneas de trabajo e investigación con el fin de incidir y mejorar la opinión que las propias pacientes tienen a cerca de la vacuna. Como limitaciones del estudio se dispone de un número reducido de pacientes y sería necesario aumentar el tamaño muestral para tener una estimación de la situación en nuestro país más aproximada.

\section{CONCLUSIÓN}

Afirmamos que el coste de la vacuna es la causa principal de rechazo a la vacunación en pacientes tratadas por patología cervical en Galicia, España. El grado de información y conocimiento sobre el VPH son determinantes para la aceptación de la vacuna, siendo esta la segunda causa de la no vacunación en nuestro estudio. Serán necesarios estudios posteriores para evaluar la eficacia de la vacunación, en mujeres fuera de los programas de vacunación sistemática, como intervención preventiva.

\section{REFERENCIAS}

1. Bosch FX. The path to eliminate cervical cancer in the world and the challenges of professional education. Vaccine 2013;31 Suppl 7: xi - xii.

2. Schiffman $\mathrm{MH}$, Bauer HM, Hoover RN, et al. Epidemiologic evidence showing that human papillomavirus infection causes most cervical intraepithelial neoplasia. J Natl Cancer Inst 1993; 85:958-64.

3. Kaufman $\mathrm{RH}$, Adam E, Icenogle J, et al. Relevance of human papillomavirus screening in management of cervical intraepithelial neoplasia. Am J Obstet Gynecol 1997; 176:87-92.

4. Limia A, Pachon I. Coverage of human papillomavirus vaccination during the first year of its introduction in Spain. Euro Surveill 2011;16:pii:19873.

5. Castellagué $X$, Muñoz N, Pitisuttithum $P$, Ferris $D$, Monsonego J, Ault $\mathrm{K}$, et al. End-of-study safety, immunogenicity, and efficacy of quadrivalent HPV (types $6,11,16,18$ ) recombinant vaccine in adult women 24-45 years of age. Br J Cancer 2011;105:28-37.

6. Muñoz N, Manalastas R Jr, Pitisuttithum P, Tresukosol D, Monsonego J, Ault K, et al. Safety, immunogenicity, and efficacy of quadrivalent human papillomavirus (types $6,11,16,18$ ) recombinant vaccine in women aged $24-45$ years: a randomised, double-blind trial. Lancet 2009;373:1949-57.

7. Descamps DSA, Garland S, Lazcano E, David MP, Hardt K, Dubin G, et al. HPV-16/18 AS04-adjuvanted vaccine efficacy in $\geq 26$-year-old women after 4-year follow-up27. International Papillomavirus Conference. 2011;O-18.06.

8. Torné Bladé A, Bayas Rodríguez JM, Castellsagué Piqu, X, Sánchez MC, García García E, Martínez Escoriza JC, et al. Vacunación frente al cáncer de cérvix en mujeres fuera de los programas de vacunación sistemática, con o sin infección por el virus del papiloma humano o lesión cervical. Encuesta de opinión y recomendaciones. Prog Obstet Ginecol 2012;55 (Suppl 1):10-31.

9. Orlando G, Fasolo M, Mazza F, Ricci E, Esposito S, Frati $\mathrm{E}$, et al. Risk of cervical HPV infection and prevalence of vaccine-type and other high-risk HPV types among sexually active teens and young women (13-26 years) enrolled in the VALHIDATE study. Hum Vaccin Immunother 2014;10(4). ppi:27682.

10. Munoz N, Bosch FX, de San José S, Herrero R, Castellsague X, Shah KV, et al. Epidemiologic classification of human papillomavirus types associated with cervical cancer. N Engl J Med 2003;348:518-27.

11. Sudenga SL, Shrestha S. Key considerations and current perspectives of epidemiological studies on human papillomavirus persistence, the intermediate phenotype to cervical cancer. Int $\mathrm{J}$ Infect Dis 2013;17:e216-e220. 
12. Davis K, Dickman ED, Ferris D, Dias JK. Human papillomavirus vaccine acceptability among parents of 10- to 15-year-old adolescents. J Low Genit Tract Dis 2004;8:188-94.

13. Hopkins TG, Wood N. Female human papillomavirus (HPV) vaccination: Global uptake and the impact of attitudes. Vaccine 2013;31:1673-9.

14. Schiller JT, Castellsagué X, Garland SM. A review of clinical trials of human papillomavirus prophylactic vaccines. Vaccine 2012;30:F123-F138.

15. Jaspers L, Budiningsih S, Wolterbeek R, Henderson FC, Peters AA. Parental acceptance of human papillomavirus (HPV) vaccination in Indonesia: a cross sectional study. Vaccine 2011;29:7785-93.
16. Chow SN, Soon R, Park JS, Pancharoen C, Qiao YL, Basu $P$, et al. Knowledge, attitudes, and communication around human papillomavirus (HPV) vaccination amongst urban Asian mothers and physicians. Vaccine 2010;28:3809-17.

17. Wong LP. Knowledge and attitudes about HPV infection, HPV vaccination, and cervical cancer among rural Southeast Asian women. Int $\mathrm{J}$ Behav Med 2011;18:105-11.

18. Basu P, Mittal S. Acceptability of human papillomavirus vaccine among the urban, affluent and educated parents of young girls residing in Kolkata, Eastern India. J Obstet Gynaecol Res 2011;37:393-401. 